Check for updates

Cite this: Phys. Chem. Chem. Phys., 2017, 19, 27580

Received 4th August 2017, Accepted 15th September 2017 DOI: $10.1039 / c 7 c p 05300 b$

rsc.li/pccp

\section{Explicit description of complexation between oppositely charged polyelectrolytes as an advantage of the random phase approximation over the scaling approach}

\author{
Artem M. Rumyantsev (D) ab and Igor I. Potemkin (D)*abc \\ A polyelectrolyte complex (PEC) of oppositely charged linear chains is considered within the Random \\ Phase Approximation (RPA). We study the salt-free case and use the continuous model assuming a \\ homogeneous distribution of the charges throughout the polyions. The RPA correction to the PEC free \\ energy is renormalized via subtraction of polyion self-energy in order to find the correlation free energy \\ of the complex. An analogous procedure is usually carried out in the case of the Debye-Hückel (DH) \\ plasma (a gas of point-like ions), where the infinite self-energy of point-like charges is subtracted from \\ the diverging RPA correction. The only distinction is that in the PEC both the RPA correction and chain \\ self-energy of connected like charges are convergent. This renormalization allows us to demonstrate \\ that the correlation free energy of the PEC is negative, as could be expected, while the scaling approach \\ postulates rather than proving the negative sign of the energy of interactions between the blobs. We \\ also demonstrate that the increasing concentration of oppositely charged polyions in the solution first \\ results in the formation of neutral globules of the PEC consisting of two polyions as soon as the \\ concentration reaches a certain threshold value, $c_{g l}$, whereas solution macroscopic phase separation \\ (precipitation of globules) occurs at a much higher concentration, $c_{\mathrm{coac}}, c_{\mathrm{coac}} \gg c_{\mathrm{gl}}$. Partitioning of \\ polyions between different states is calculated and analytical dependencies of $c_{g l}$ and $c_{\text {coac }}$ on the \\ polyion length, degree of ionization and solvent polarity are found.
}

\section{Introduction}

Correlation effects are of major importance in many charged systems, especially if they comprise macro- and/or multivalent co- and counterions, ${ }^{1}$ which control the strength and character of electrostatic interactions. In addition to the valency of the ions, phase behavior of charged liquids (solutions of colloidal particles, polyelectrolytes (PEs), etc.) depends on relative "asymmetry" of co- and counterions. For example, bulky macroions (like charged colloidal particles, microgels ${ }^{2,3}$ or linear $\mathrm{PEs}^{4}$ ) with small (pointlike) monovalent counterions can be attributed to strongly "asymmetric" charged solutions where high entropic mobility of the counterions protects complete local neutralization of the system and formation of a condensed phase. Therefore, the presence of charged groups in such asymmetric systems can promote solubility of the macroions. In contrast, in charge "symmetric"

\footnotetext{
${ }^{a}$ Physics Department, Lomonosov Moscow State University, 119991 Moscow, Russian Federation.E-mail: igor@polly.phys.msu.ru

${ }^{b}$ DWI - Leibniz Institute for Interactive Materials, Aachen 52056, Germany

${ }^{c}$ National Research South Ural State University, Chelyabinsk 454080,

Russian Federation
}

systems, like solutions of oppositely charged PEs,${ }^{5-9}$ condensation of the macroions occurs in the isoelectric point (total charge of the macrions is equal to zero). Therefore, the electrostatic attraction decreases the solubility of the macroions in the "symmetric" system. ${ }^{10-12}$ In the intermediate cases of the asymmetry or beyond the isoelectric point, the interplay between attractive and repulsive electrostatic interactions is responsible for the clustering of the macroions in soluble aggregates. In many cases, solubility of the aggregates is provided by the effect of overcharging (charge inversion): ${ }^{13-16}$ the total charge of the complex becomes opposite to the bare charge of the macroions.

The spatial charge distribution in the "asymmetric" systems is essentially inhomogeneous. It allows developing mean-field theories (Poison-Boltzmann or multi-zone approximation) like for dilute solutions of PE micelles ${ }^{17-20}$ or microgels. ${ }^{21}$ On the other hand, the inhomogeneous charge distribution means strong spatial charge fluctuations which are equivalent to the strong charge fluctuations at a certain spatial coordinate evolving over time as long as the ergodic hypothesis is valid. Therefore, calculations of the fluctuation contribution to the free energy in such systems are sophisticated. In contrast, charge fluctuations are much smaller in the "symmetric" solutions and the mean-field treatment is 
not able to describe the Coulomb interactions properly (they are equal to zero at this level). The electrostatics due to the small charge fluctuations can be taken into account within the framework of the Random Phase Approximation (RPA), which is a linear response approximation analogous to the one-loop approximation used in quantum field theory.

Over 90 years ago, Debye and Hückel (DH) have considered the problem of electrolyte solutions (the simplest "symmetric" system) demonstrating deviations from the ideal gas behaviour. ${ }^{22}$ Using the Poison-Boltzmann equation linearization, they have calculated corrections to the osmotic pressure and to the free energy of the ideal gas, both are negative. However, independently of the valency of the ions, their condensation or ion pair formation cannot be described within such an approach. The condition for linearization is that the energy of the electrostatic interactions between the ions is much smaller than the energy of their thermal motion. Three decades later, the first theoretical attempt to describe saline solutions of oppositely charged PEs was undertaken by Overbeek and Voorn. ${ }^{23}$ They have treated non-electrostatic interactions within the Flory-Huggins lattice theory, while Coulomb interactions between all charges of the system (mobile lowmolecular-weight ions and charged groups of the chains) have been described using the DH expression. Thus, connectivity of the charged groups into the chains was neglected.

In the late 1980s in pioneer works of Borue and Erukhimovich, the RPA was applied to semidilute PE solutions and complexes, where the charge connectivity into the chain was taken into consideration by the addition of the polyion conformational entropy term to the free energy functional. ${ }^{24,25}$ The beauty of the RPA for the oppositely charged PEs is that the condensation of the chains (complex formation) can properly be described at this level because polymer chains are known to be "poor in entropy": even small attraction between charged groups is able to overcome the entropic penalty due to the complex formation. Possibility of microphase separation in semidilute PE solutions and mixtures of oppositely charged polyions was predicted within the framework of this approach. ${ }^{24,26-28}$ Later on, similar theory was applied for the description of PE multilayers, ${ }^{29}$ polyampholytes ${ }^{30}$ and overcharged interpolyelectrolyte complexes. ${ }^{31,32}$ Further development of the RPA included consideration of ion pairing, ${ }^{28,33,34}$ short wavelength integration cutoff, ${ }^{33,35}$ hard-core and finite-size ion effects. ${ }^{33,34,36,37}$ In our group, fluctuations of polymer and charge densities were examined independently (discrete charge distribution), which allowed getting a general expression for the free energy unifying different limiting cases. ${ }^{38}$ The RPA theory for rod-like polymer chains capable of liquid-crystalline ordering was developed in ref. 39-42. Treatment of single PE chain trapping own counterions as one-component plasma (i.e. point-like charges immersed into homogeneously and oppositely charged background) is also based on the RPA. ${ }^{35,43-46}$ Castelnovo and Joanny, ${ }^{47}$ and then Fredrickson and co-workers ${ }^{48}$ extended the fluctuation theory of complexation beyond the RPA.

Recently account for fluctuations by means of liquid state theory approaches allowed describing solutions ${ }^{49}$ and complexes ${ }^{50}$ of highly charged PEs. In the framework of this method it was theoretically shown that low-molecular-weight salt ions prefer a supernatant rather than a coacervate, ${ }^{50}$ in contrast to predictions of Vroon-Overbeek theory that neglects both connectivity of PE charges and excluded volume interactions. ${ }^{37,51,52}$ This finding on salt ion partitioning being in principal agreement with experiment was later confirmed using computer simulations. ${ }^{53,54}$

In the present paper, we propose a procedure of physically motivated renormalization of the RPA results for the symmetric PEC allowing us to distinguish the correlation (free) energy of the PEC and self-energy of polyions in the salt-free case. Proper renormalization makes possible to find partitioning of polyions between different states (non-aggregated chains, neutral PEC globules and coacervates) in a stoichiometric mixture of polyanions and polycations. Threshold concentrations of globule formation and macroscopic phase separation are found analytically.

The model with a charge continuously smeared throughout the flexible chain is used for the theoretical treatment of the PEC. ${ }^{24,55}$ The RPA correction to the free energy is known to be convergent within the framework of this model. ${ }^{29-32}$ However, the knowledge of this positive expression, which allows us to find the electrostatic contribution to the osmotic pressure and the polymer volume fraction in the complex properly, is not enough for calculation of the correlation (free) energy. Nonzero self-energy of the chains caused by connectivity of the charged groups has to be calculated and subtracted from the RPA results. The self-energy takes into account repulsive interactions of the charges in each individual chain. Due to the subtraction procedure, the correlation energy, which corresponds to the attraction between oppositely charged chains, occurs to be negative as one would expect. In contrast to the scaling approach, where the interaction energy between the blobs was postulated to be negative, ${ }^{56}$ the RPA proposes a straightforward way for calculation of the correlation free energy defining the numerical factor as soon as one deals with the polymers in a $\Theta$-solvent. In particular, the knowledge of the self-energy allows quantifying the association of single chains into a complex (calculation of the critical concentration of association).

Here it is necessary to mention that initially a renormalization procedure of the RPA results for PE solutions based on the subtraction of chains self-energy was proposed by Mahdi and Olvera de la Cruz who motivated it primarily by mathematical convenience. $^{36}$ Lately Shen and Wang have explicitly distinguished self-energy of polyions in PE solution and found solution correlation energy via subtraction of the chain selfenergy from the formal field-theoretic result. ${ }^{57}$ In the present paper, we apply the same renormalization procedure to the case of the PEC. It makes possible to argue for the first time the negative sign of the complex correlation energy within the RPA.

In order to make the calculation of the correlation free energy in the case of PECs more lucid and clear, we use analogy with wellknown DH plasma wherein subtraction of infinite self-energy of point-like ions from the diverging RPA result is necessary to get a finite negative expression for the correlation free energy. An analogy between correlation functions in the case of the PE complex and the DH plasma also occurs to be very useful.

The paper is organized as follows. In the next section the DH plasma is discussed in detail, first without and then with 
volume interactions, and well-known results for ion-ion correlation functions and correlation free energy of plasma are reproduced. In Section 3, we move on to the case of the PEC and propose a renormalization procedure of the RPA free energy correction. It allows us to obtain the correlation free energy of the complex. Section 4 deals with the calculation of the critical concentrations of PEC formation and coacervation. Closed-form expressions for these concentrations are found.

\section{Debye-Hückel plasma}

We consider a neutral plasma consisting of oppositely charged ions, each of the size $a$ and volume $a^{3}$. Let the average volume fractions of positively and negatively charged ions are equal: $\left\langle n_{+}\right\rangle=\left\langle n_{-}\right\rangle=n_{0}$. Here $n_{0}$ is dimensionless, so that the concentration of each type of the ions equals $n_{0} / a^{3}$. Neglecting short-range interactions between the ions under the condition of a small concentration, $n_{0} \ll 1$, one gets the classical case of the DH plasma of point-like charges. The total free energy is a sum of two terms:

$$
F_{\text {tot }}\left\{n_{+}(r), n_{-}(r)\right\}=F_{\text {tr }}+F_{\text {el-st }}
$$

the ideal gas entropy

$$
\frac{F_{\mathrm{tr}}}{k_{\mathrm{B}} T}=\frac{1}{a^{3}} \int\left[n_{+}(\mathbf{r}) \ln \left(\frac{n_{+}(\mathbf{r})}{e}\right)+n_{-}(\mathbf{r}) \ln \left(\frac{n_{-}(\mathbf{r})}{e}\right)\right] \mathrm{d}^{3} r
$$

and the energy of long-range Coulomb interactions between the ions

$$
\frac{F_{\mathrm{el}-\mathrm{st}}}{k_{\mathrm{B}} T}=\frac{l_{\mathrm{b}}}{2 a^{6}} \int \frac{\left(n_{+}(\mathbf{r})-n_{-}(\mathbf{r})\right)\left(n_{+}\left(\mathbf{r}^{\prime}\right)-n_{-}\left(\mathbf{r}^{\prime}\right)\right)}{\left|\mathbf{r}-\mathbf{r}^{\prime}\right|} \mathrm{d}^{3} r \mathrm{~d}^{3} r^{\prime} .
$$

Here, $l_{\mathrm{b}}=e^{2} /\left(\varepsilon k_{\mathrm{B}} T\right)$ is the Bjerrum length.

Correlated electrostatic attraction makes the osmotic pressure of the DH plasma lower than that of the ideal gas. The RPA can be used in order to find the free energy correction to the ideal gas term and to calculate the density-density correlation functions of the DH plasma. The concentration fluctuations are defined as $\delta n_{k}(\mathbf{r})=n_{k}(\mathbf{r})-n_{0}$, and Fourier transforms are given by

$$
\begin{gathered}
\delta n_{k}(\mathbf{q})=\int \delta n_{k}(\mathbf{r}) \mathrm{e}^{\mathrm{i} \mathbf{q r} \mathrm{d}^{3} r} \\
\delta n_{k}(\mathbf{r})=\frac{1}{(2 \pi)^{3}} \int \delta n_{k}(\mathbf{q}) \mathrm{e}^{-\mathrm{i} \mathbf{q r}} \mathrm{d}^{3} q
\end{gathered}
$$

where $k=+,-$. Deviation of the free energy functional from the mean-field value, $F_{\mathrm{MF}} / k_{\mathrm{B}} T=2 V n_{0} \ln \left(n_{0} / e\right) / a^{3}$, in the Gaussian (square) approximation reads:

$$
\begin{aligned}
\frac{\delta F_{\text {tot }}}{k_{\mathrm{B}} T}= & \frac{1}{2(2 \pi)^{3} n_{0} a^{3}} \int\left[\left|\delta n_{+}(\mathbf{q})\right|^{2}+\left|\delta n_{-}(\mathbf{q})\right|^{2}\right] \mathrm{d}^{3} q \\
& +\frac{2 \pi l_{\mathrm{b}}}{(2 \pi)^{3} a^{6}} \int \frac{\left|\delta n_{+}(\mathbf{q})-\delta n_{-}(\mathbf{q})\right|^{2}}{q^{2}} \mathrm{~d}^{3} q
\end{aligned}
$$

where $V$ is the total system volume. Owing to the following representation of the free energy fluctuations

$$
\frac{\delta F_{\text {tot }}}{k_{\mathrm{B}} T}=\frac{1}{2} \int \frac{G_{k l}{ }^{-1}(\mathbf{q})}{(2 \pi)^{3}} \delta n_{k}(\mathbf{q}) \delta n_{l}(-\mathbf{q}) \mathrm{d}^{3} q
$$

and introducing the Debye radius $r_{\mathrm{D}}{ }^{2}=a^{3} /\left(8 \pi l_{\mathrm{b}} n_{0}\right)$, one can obtain the direct matrix of the plasma correlation functions in the Fourier space:

$$
G_{k l}(\mathbf{q})=\frac{n_{0} a^{3}}{\left(1+r_{\mathrm{D}}^{2} q^{2}\right)}\left(\begin{array}{cc}
\frac{1}{2}+r_{\mathrm{D}}^{2} q^{2} & \frac{1}{2} \\
\frac{1}{2} & \frac{1}{2}+r_{\mathrm{D}}^{2} q^{2}
\end{array}\right)
$$

The spherically symmetric density-density correlation functions $G_{k l}(r)=\left\langle\delta n_{k}(0) \cdot \delta n_{l}(r)\right\rangle$ are calculated using the inverse Fourier transforms and take the form

$$
\begin{gathered}
G_{++}(r)=G_{--}(r)=n_{0} a^{3} \delta(\mathbf{r})-l_{\mathrm{b}} n_{0}^{2} \frac{\mathrm{e}^{-r / r_{\mathrm{D}}}}{r} \\
G_{+-}(r)=G_{-+}(r)=l_{\mathrm{b}} n_{0}{ }^{2} \frac{\mathrm{e}^{-r / r_{\mathrm{D}}}}{r}
\end{gathered}
$$

The second set of the correlation functions, $G_{+-}=G_{-+}$, is caused only by the electrostatic attraction between the oppositely charged ions: it is equal to zero at $l_{\mathrm{b}}=0$. For this reason it can be designated as $G_{+-}=G_{\text {corr }}$. The first set of correlation functions, $G_{++}=G_{--}$, should be divided into two parts: $G_{++}=G_{\text {self }}-G_{\text {corr }}$. The first nonvanishing at the $l_{\mathrm{b}}=0$ part, $G_{\text {self }}=n_{0} a^{3} \delta(\mathbf{r})$ is known as a correlation function of the neutral ideal gas while the second part, $-G_{\text {corr }}$, should be ascribed to the repulsion of like charges. Note that both $G_{\text {corr }}$ and $G_{\text {self }}$ are positive at any values of $r$.

The correction to the electrostatic energy caused by ion-ion correlations (density fluctuations) can be calculated as follows:

$$
\frac{\delta U_{\mathrm{el}-\mathrm{st}}}{k_{\mathrm{B}} T}=\left\langle\frac{l_{\mathrm{b}}}{2 a^{6}} \int \frac{\left(\delta n_{+}(\mathbf{r})-\delta n_{-}(\mathbf{r})\right)\left(\delta n_{+}\left(\mathbf{r}^{\prime}\right)-\delta n_{-}\left(\mathbf{r}^{\prime}\right)\right)}{\left|\mathbf{r}-\mathbf{r}^{\prime}\right|} \mathrm{d}^{3} r \mathrm{~d}^{3} r^{\prime}\right\rangle,
$$

where the angle brackets denote the thermodynamic averaging. Owing to the expression $\left\langle\delta n_{k}(\mathbf{r}) \delta n_{l}\left(\mathbf{r}^{\prime}\right)\right\rangle=G_{k l}\left(\left|\mathbf{r}-\mathbf{r}^{\prime}\right|\right)$ and denoting $R=|\mathbf{R}|=\left|\mathbf{r}-\mathbf{r}^{\prime}\right|$, one can calculate

$$
\begin{aligned}
\frac{\delta U_{\mathrm{el}-\mathrm{st}}}{k_{\mathrm{B}} T} & =\frac{4 \pi V l_{\mathrm{b}}}{a^{6}} \int_{0}^{\infty}\left(G_{++}(R)-G_{+-}(R)\right) R \mathrm{~d} R \\
& =\frac{4 \pi V l_{\mathrm{b}}}{a^{6}} \int_{0}^{\infty}\left(G_{\text {self }}(R)-2 G_{\mathrm{corr}}(R)\right) R \mathrm{~d} R
\end{aligned}
$$

Thus, the self-energy of point-like ions and the energy of interaction between the ions are given by

$$
\begin{gathered}
\frac{U_{\text {self }}}{k_{\mathrm{B}} T}=\frac{4 \pi V l_{\mathrm{b}}}{a^{6}} \int_{0}^{\infty} G_{\text {self }}(R) R \mathrm{~d} R \\
\frac{U_{\text {corr }}}{k_{\mathrm{B}} T}=-\frac{8 \pi V l_{\mathrm{b}}}{a^{6}} \int_{0}^{\infty} G_{\text {corr }}(R) R \mathrm{~d} R
\end{gathered}
$$

It is well-known that the self-energy of point-like charge diverges:

$$
\frac{U_{\text {self }}}{k_{\mathrm{B}} T}=\frac{V l_{\mathrm{b}} n_{0}}{a^{3}} \int_{0}^{\infty} \frac{\delta(R)}{R} \mathrm{~d} R \rightarrow \infty
$$

where the delta function in the spherical coordinates was substituted, $\delta(R)=\delta(\mathbf{R}) /\left(4 \pi R^{2}\right)$. On the other hand, the correlation 
internal energy is finite:

$$
\frac{U_{\text {corr }}}{k_{\mathrm{B}} T}=-\frac{8 \pi V l_{\mathrm{b}}^{2} n_{0}^{2} r_{\mathrm{D}}}{a^{6}}=-\frac{V}{8 \pi} \frac{1}{r_{\mathrm{D}}^{3}}
$$

The energy is negative and decreases with the increase of the concentration $n_{0}$. It means that the electrostatic interactions are attractive. To pass to the Helmholtz free energy correction, one can apply the relation between thermodynamic potentials

$$
U=-T^{2} \frac{\partial}{\partial T}\left(\frac{F}{T}\right)
$$

As a result, the free energy density correction reads

$$
\frac{F_{\text {corr }}}{k_{\mathrm{B}} T V}=-\frac{1}{12 \pi r_{\mathrm{D}}^{3}}+A_{1}
$$

and constant $A_{1}=0$ because the condition $F_{\text {corr }}=\left(F_{\text {tot }}-F_{\text {id }}\right) \rightarrow 0$ at $T \rightarrow \infty$ should be satisfied, $F_{\text {id }}$ being the free energy of neutral ideal gas equal to $F_{\mathrm{MF}}{ }^{58}$

Another way to get the correlation free energy correction is based on the calculation of Gaussian functional integrals

$$
\begin{aligned}
\frac{F_{\mathrm{RPA}}}{k_{\mathrm{B}} T}= & -\ln \int \mathscr{D} \delta n_{+} \mathscr{D} \delta n_{-} \exp \left(-\frac{\delta F_{\mathrm{tot}}\left\{\delta n_{+}, \delta n_{-}\right\}}{k_{\mathrm{B}} T}\right) \\
& +\ln \int \mathscr{D} \delta n_{+} \mathscr{D} \delta n_{-} \exp \left(-\left.\frac{\delta F_{\mathrm{tot}}\left\{\delta n_{+}, \delta n_{-}\right\}}{k_{\mathrm{B}} T}\right|_{l_{\mathrm{b}}=0}\right)
\end{aligned}
$$

In order to calculate the integrals, quadratic form

$$
G_{k l}{ }^{-1}(q)=\frac{1}{n_{0} a^{3}}\left(\begin{array}{cc}
1+\frac{1}{2 r_{\mathrm{D}}^{2} q^{2}} & -\frac{1}{2 r_{\mathrm{D}}^{2} q^{2}} \\
-\frac{1}{2 r_{\mathrm{D}}^{2} q^{2}} & 1+\frac{1}{2 r_{\mathrm{D}}^{2} q^{2}}
\end{array}\right)
$$

should be diagonalized. After transformations, one gets the following expression

$$
\begin{aligned}
& \frac{F_{\mathrm{RPA}}}{k_{\mathrm{B}} T}=\frac{1}{2} \frac{V}{(2 \pi)^{3}} \int \ln \left(\frac{\operatorname{det}\left[G_{k l}^{-1}(q)\right]}{\left.\operatorname{det}\left[G_{k l} l^{-1}(q)\right]\right|_{l_{\mathrm{b}}=0}}\right) \mathrm{d}^{3} q \\
& =\frac{1}{2} \frac{V}{(2 \pi)^{3}} \int \ln \left(1+\frac{1}{r_{\mathrm{D}}^{2} q^{2}}\right) \mathrm{d}^{3} q
\end{aligned}
$$

diverging at $q \rightarrow \infty$, i.e. at $r \rightarrow 0$. This divergence is a consequence of infinite self-energy of point-like charges. $F_{\mathrm{RPA}}$ includes both the self- and the interaction energies, $F_{\mathrm{RPA}}=F_{\text {self }}+F_{\text {corr }}$. The self-energy is given by eqn (15) and can be rewritten using Fourier transform:

$$
\frac{F_{\text {self }}}{k_{\mathrm{B}} T}=\frac{1}{2} \frac{V}{(2 \pi)^{3}} \int \frac{1}{r_{\mathrm{D}}^{2} q^{2}} \mathrm{~d}^{3} q
$$

Finally, the converging result for the correlation free energy

$$
\frac{F_{\text {corr }}}{k_{\mathrm{B}} T V}=\frac{1}{2} \int\left[\ln \left(1+\frac{1}{r_{\mathrm{D}}^{2} q^{2}}\right)-\frac{1}{r_{\mathrm{D}^{2} q^{2}}}\right] \frac{\mathrm{d}^{3} q}{(2 \pi)^{3}}=-\frac{1}{12 \pi r_{\mathrm{D}}^{3}}
$$

coincides with one deduced above. It is also worth noting that considering ions of non-zero radius and finite charge density function instead of point-like ions with $\delta$-function type charge distribution, one can avoid all energy divergences. ${ }^{59}$
As we mentioned in Introduction, the above calculations of the correlation (free) energy are valid when this correction is small as compared to the energy of thermal motion, $\left|F_{\text {corr }}\right| \ll\left|F_{\text {tr }}\right|$. This condition is fulfilled at a low concentration of the ions, $n_{0} \ll\left(a / l_{\mathrm{b}}\right)^{3}$.

\subsection{Debye-Hückel plasma with volume interactions}

In order to take into account volume interactions of the plasma ions, we can use the virial expansion and the following term should be added to the total free energy

$$
\frac{F_{\mathrm{vol}}}{k_{\mathrm{B}} T}=\frac{1}{a^{3}} \int\left[B\left(n_{+}(\mathbf{r})+n_{-}(\mathbf{r})\right)^{2}+C\left(n_{+}(\mathbf{r})+n_{-}(\mathbf{r})\right)^{3}\right] \mathrm{d}^{3} r
$$

Here $B$ and $C$ are the second and the third dimensionless virial coefficients. After expansion of the total free energy functional into the series in powers of the density fluctuations, the Fourier transforms of the correlation functions take the form:

$$
\begin{aligned}
& G_{k l}(q) \\
& =\frac{n_{0} a^{3}}{\left(1+r_{\mathrm{D}}^{2} q^{2}\right)(1+\mu)}\left(\begin{array}{cc}
\frac{1}{2}+\left(1+\frac{\mu}{2}\right) r_{\mathrm{D}}^{2} q^{2} & \frac{1}{2}-\frac{\mu}{2} r_{\mathrm{D}}^{2} q^{2} \\
\frac{1}{2}-\frac{\mu}{2} r_{\mathrm{D}}^{2} q^{2} & \left(1+\frac{\mu}{2}\right)+r_{\mathrm{D}}^{2} q^{2}
\end{array}\right)
\end{aligned}
$$

were $\mu=4 n_{0}\left(B+6 C n_{0}\right)$. The value $\mu=0$ corresponds to the case without volume interactions, and the matrix coincides with the matrix 8. The correlation functions read

$$
\begin{array}{r}
G_{++}(r)=G_{--}(r) \\
=\left(1-\frac{\mu}{2(1+\mu)}\right) n_{0} a^{3} \delta(\mathbf{r})-l_{\mathrm{b}} n_{0}{ }^{2} \frac{\mathrm{e}^{-r / r_{\mathrm{D}}}}{r} \\
G_{+-}(r)=G_{-+}(r)=-\frac{\mu}{2(1+\mu)} n_{0} a^{3} \delta(\mathbf{r})+l_{\mathrm{b}} n_{0}{ }^{2} \frac{\mathrm{e}^{-r / r_{\mathrm{D}}}}{r}
\end{array}
$$

Due to the RPA linearity, the correlation functions can be represented as a sum of (i) the ideal gas self-correlation function, $G_{\text {self }}=n_{0} a^{3} \delta(\mathbf{r})$, (ii) the term caused by the volume interactions, $G_{\text {vol }}=-\mu n_{0} a^{3} \delta(\mathbf{r}) /(2+2 \mu)$, and (iii) the term $G_{\text {corr }}=l_{\mathrm{b}} n_{0}{ }^{2} \mathrm{e}^{-r / r_{\mathrm{D}}} / r$ induced by the electrostatic interactions:

$$
\begin{gathered}
G_{++}=G_{\text {self }}+G_{\mathrm{vol}}-G_{\text {corr }} \\
G_{+-}=G_{\mathrm{vol}}+G_{\text {corr }}
\end{gathered}
$$

The terms $G_{\text {self }}$ and $G_{\text {corr }}$ coincide with those defined above for the DH plasma without volume interactions. $G_{\text {vol }}$ term vanishes when the triple repulsion compensates pairwise attraction, $B=-6 C n_{0}$. Following eqn (19), one can calculate the RPA correction to the free energy caused by the Coulomb interaction. Despite the presence of the volume interactions, the result for $F_{\mathrm{RPA}}$ coincides with eqn (21). Thus, the self-energy given by eqn (13) should be subtracted from $F_{\mathrm{RPA}}$ to find the correlation energy, and the latter coincides with correlation energy for the $\mathrm{DH}$ plasma without volume interactions, $F_{\text {corr }} / k_{\mathrm{B}} T V=-1 /\left(12 \pi r_{\mathrm{D}}^{3}\right)$. Independence of $F_{\mathrm{RPA}}$ and $F_{\text {corr }}$ of the volume interactions should be attributed to the linearity of the RPA. 
Note that eqn (11)-(14) remain valid in the case of non-zero volume interactions as well, so that definitions of the self-energy and the correlation energy are the same. Indeed, relationship $G_{++}-G_{+-}=G_{\text {self }}-2 G_{\text {corr }}$ is satisfied despite $G_{\text {vol }} \neq 0$. In the general case, the term caused purely by the electrostatic interactions should be defined as

$$
G_{\text {corr }}=\left.\left(G_{+-}\right)\right|_{B=C=0}
$$

In the next section we apply the proposed approach to describe complex formation between oppositely charged PE chains.

\section{Interpolyelectrolyte complex}

Let us consider a complex of two oppositely charged linear PEs in a salt-free, $\Theta$-solvent. For the sake of simplicity, we study the case of a symmetric complex, i.e. $f_{+}=f_{-}=f$ are the fractions of charged groups in these polyions, and $\Phi_{+}$and $\Phi_{-}$are their average polymer volume fractions in complex, respectively. The complex electric neutrality is provided by condition $\Phi_{+}=\Phi_{-}=\Phi_{\text {tot }} / 2$, where $\Phi_{\text {tot }}$ is the total polymer volume fraction within the complex. The lengths of polycations and polyanions are also assumed to be equal, $N_{+}=N_{-}=N$ (see ref. 60 for the asymmetric case). We study the classical model proposed by Borue and Erukhimovich which assumes that electric charge is continuously smeared throughout the chains. ${ }^{24,25}$

The total free energy in $k_{\mathrm{B}} T$ units takes the form:

$$
F_{\text {tot }}\left\{\Phi_{+}(r), \Phi_{-}(r)\right\}=F_{\text {tr }}+F_{\text {conf }}+F_{\text {el-st }}+F_{\text {vol }}
$$

The first term is responsible for the translational entropy of polymer chains:

$$
\frac{F_{\mathrm{tr}}}{k_{\mathrm{B}} T}=\frac{1}{N a^{3}} \int\left[\Phi_{+}(\mathbf{r}) \ln \left(\frac{\Phi_{+}(\mathbf{r})}{e}\right)+\Phi_{-}(\mathbf{r}) \ln \left(\frac{\Phi_{-}(\mathbf{r})}{e}\right)\right] \mathrm{d}^{3} r
$$

The second term is the conformational entropy of polyions ${ }^{61,62}$

$$
\frac{F_{\text {conf }}}{k_{\mathrm{B}} T}=\frac{1}{6 a} \int\left[\left(\nabla \sqrt{\Phi_{+}(\mathbf{r})}\right)^{2}+\left(\nabla \sqrt{\Phi_{-}(\mathbf{r})}\right)^{2}\right] \mathrm{d}^{3} r
$$

with $a$ being the length of the statistical segment. Deduced independently by Edwards and later by Lifshitz, it takes into account the entropy cost of the polymer non-uniform distribution. The case of flexible polymer chains is under consideration, so that $a^{3}$ is of the order of the segment volume. The Coulomb energy is given by

$$
\frac{F_{\mathrm{el}-\mathrm{st}}}{k_{\mathrm{B}} T}=\frac{l_{\mathrm{b}} f^{2}}{2 a^{6}} \int \frac{\left(\Phi_{+}(\mathbf{r})-\Phi_{-}(\mathbf{r})\right)\left(\Phi_{+}\left(\mathbf{r}^{\prime}\right)-\Phi_{-}\left(\mathbf{r}^{\prime}\right)\right)}{\left|\mathbf{r}-\mathbf{r}^{\prime}\right|} \mathrm{d}^{3} r \mathrm{~d}^{3} r^{\prime}
$$

We restrict our consideration to the case of weakly charged PEs, $f \ll 1$, and a rather polar solvent, $u \sim 1$, so that effects of crosschain ion pairing ${ }^{63-65}$ and attraction of resultant dipoles ${ }^{66-68}$ can be neglected. Finally, the volume interactions, namely triple repulsive interactions between the segments in the $\Theta$-solvent, are taken into account within the last term of the total free energy

$$
\frac{F_{\mathrm{vol}}}{k_{\mathrm{B}} T}=\frac{1}{a^{3}} \int C\left(\Phi_{+}(\mathbf{r})+\Phi_{-}(\mathbf{r})\right)^{3} \mathrm{~d}^{3} r
$$

with $C$ being the dimensionless third virial coefficient. The mean-field value of the total free energy of the neutral complex takes the form:

$$
\frac{F_{\mathrm{tot}}^{\mathrm{MF}}}{k_{\mathrm{B}} T} \frac{a^{3}}{V}=C \Phi_{\mathrm{tot}}{ }^{3}+\frac{\Phi_{\mathrm{tot}}}{N} \ln \left(\frac{\Phi_{\mathrm{tot}}}{2 e}\right)
$$

where the second term is negligible at high $N$, i.e. the volume interactions dominate over the chain translational entropy. Thus, the repulsive interactions make the complex unstable. The complex formation cannot be described at the mean-field level because the latter neglects the electrostatic attractions between charged chains. At the same time, the complex formed by oppositely charged PEs is globular, and weak fluctuation within the globule provides applicability of the RPA (see ref. 25 for detailed discussion).

Expansion of the free energy functional into the series in powers of the polymer density fluctuations allows calculating the inverse matrix of correlation functions

$$
\begin{aligned}
& G_{k l}{ }^{-1}(q)=\frac{q^{2}}{6 a \Phi_{\mathrm{tot}}} \\
& \times\left(\begin{array}{cc}
1+\frac{1}{r_{\mathrm{c}}^{2} q^{2}}+\frac{1}{l_{\mathrm{vol}}^{2} q^{2}}+\frac{1}{2 \xi^{4} q^{4}} & \frac{1}{l_{\mathrm{vol}}^{2} q^{2}}-\frac{1}{2 \xi^{4} q^{4}} \\
\frac{1}{l_{\mathrm{vol}}^{2} q^{2}}-\frac{1}{2 \xi^{4} q^{4}} & 1+\frac{1}{r_{\mathrm{c}}^{2} q^{2}}+\frac{1}{l_{\mathrm{vol}}^{2} q^{2}}+\frac{1}{2 \xi^{4} q^{4}}
\end{array}\right)
\end{aligned}
$$

with $r_{\mathrm{c}}=a \sqrt{N} / \sqrt{12}$ being the correlation radius of the ideal polymer chain (i.e. the Gaussian chain size), $l_{\mathrm{vol}}=a /\left(6 \sqrt{C} \Phi_{\mathrm{tot}}\right)$ being the correlation radius of infinite neutral chain caused solely by the volume interactions and $\xi=a /\left(48 \pi u f^{2} \Phi_{\text {tot }}\right)^{1 / 4}$ being the screening radius of the electrostatic interactions in $\mathrm{PE}$ complex; $u=l_{\mathrm{b}} / a$ is the dimensionless Bjerrum length. The RPA correction to the free energy of the PEC can be found in a similar way to that for the DH plasma (see eqn (19) and (21)):

$$
\frac{F_{\mathrm{RPA}}}{k_{\mathrm{B}} T}=\frac{1}{2} \frac{V}{(2 \pi)^{3}} \int \ln \left(1+\frac{1}{q^{2} \xi^{4}\left(q^{2}+{r_{\mathrm{c}}}^{-2}\right)}\right) \mathrm{d}^{3} q
$$

and this integral converges at any $r_{\mathrm{c}}$ and any finite $\xi$ (infinite $\xi$ corresponds to the case of neutral polymers, $f=0$ ). Thus, one gets

$$
\frac{F_{\mathrm{RPA}}}{k_{\mathrm{B}} T V}=\frac{1}{12 \pi r_{\mathrm{c}}{ }^{3}}+\frac{1}{6 \sqrt{2} \pi \xi^{3}}\left(1-\frac{\xi^{2}}{r_{\mathrm{c}}^{2}}\right) \sqrt{1+\frac{\xi^{2}}{2 r_{\mathrm{c}}^{2}}} .
$$

$F_{\mathrm{RPA}}$ is always positive as soon as the complex is formed $\left(\xi<r_{\mathrm{c}}\right)$. In order to get the correlation free energy of the PEC, the selfenergy of polyions should be subtracted from the RPA results. Following an analogy between the PEC and the DH plasma, the correlation functions should be divided into the parts related to connectivity of monomer units into the chain, $G_{\text {self }}$, electrostatic, $G_{\text {corr }}$, and volume, $G_{\mathrm{vol}}$, interactions:

$$
\begin{gathered}
G_{++}=G_{\text {self }}+G_{\mathrm{vol}}-G_{\text {corr }} \\
G_{+-}=G_{\mathrm{vol}}+G_{\text {corr }}
\end{gathered}
$$


The term $G_{\text {self }}$ appears only in the correlation functions of like charges as a consequence of their connectivity into a chain. Indeed, the charges of opposite signs belong to different chains and are not linked with each other by the covalent bonds.

In order to find the correlation free energy and the selfenergy, one should explicitly calculate these components of the correlation functions:

$$
\begin{gathered}
G_{\text {self }}(r)=\frac{3 a \Phi_{\text {tot }}}{2 \pi r} \mathrm{e}^{-r / r_{\mathrm{c}}} \\
G_{\mathrm{vol}}(r)=\frac{3 a \Phi_{\mathrm{tot}}}{4 \pi r}\left[\mathrm{e}^{-r / l_{\mathrm{corr}}}-\mathrm{e}^{-r / r_{\mathrm{c}}}\right] \\
G_{\mathrm{corr}}(r)=\frac{3 a \Phi_{\mathrm{tot}}}{4 \pi r}\left[\mathrm{e}^{-r / r_{\mathrm{c}}}-\frac{\xi_{+} \xi_{-}}{\xi^{2}} \cos \left(\frac{r}{\sqrt{2} \xi_{-}}+\delta\right) \mathrm{e}^{-r / \sqrt{2} \xi_{+}}\right]
\end{gathered}
$$

Here $l_{\text {corr }}$ is known to be the correlation radius of neutral polymer solution

$$
l_{\text {corr }}^{2}=\frac{1}{r_{\mathrm{c}}^{-2}+2 l_{\mathrm{vol}}{ }^{-2}}=\frac{a^{2}}{12\left(1 / N+6 C \Phi_{\mathrm{tot}^{2}}{ }^{2}\right.},
$$

with the radii $\xi_{ \pm}=\xi / \sqrt{1 \mp \xi^{2} / 2 r_{\mathrm{c}}^{2}}$ and dimensionless $\delta=\arctan \left(\xi_{+} \xi_{-} / 2 r_{\mathrm{c}}{ }^{2}\right)$. Note that the electrostatic interactions vanish at $l_{\mathrm{b}}=0$, and $G_{\mathrm{corr}}=0$. Similarly, $G_{\mathrm{vol}}=0$ in the absence of volume interactions, i.e. at $C=0 . G_{\text {self }}$ is known to be the Ornstein-Zernike type correlation function of a single Gaussian chain and is equal to the correlation function in the solution of the ideal chains. ${ }^{69}$

Using relationship similar to eqn (13) and introducing additional multiplier $f^{2}$ for the transition from the polymerpolymer to charge-charge correlation function, one obtains

$$
\frac{U_{\text {self }}}{k_{\mathrm{B}} T}=6 f^{2} N \frac{l_{\mathrm{b}} r_{\mathrm{c}}}{a^{2}} \mathscr{N}_{\text {chains }}=\sqrt{3} u f^{2} N^{3 / 2} \mathscr{N}_{\text {chains }}
$$

where $\mathscr{N}_{\text {chains }}=V \Phi_{\text {tot }} /\left(a^{3} N\right)$ is the total number of polymer chains (both polycations and polyanions) in the system.

Since $u \sim 1 / T$, the free and the internal electrostatic energies coincide, $F_{\text {self }}=U_{\text {self }}$, and the density of the self-energy can be represented as follows:

$$
\frac{F_{\text {self }}}{k_{\mathrm{B}} T} \frac{a^{3}}{V}=\sqrt{3} u f^{2} \sqrt{N} \Phi_{\mathrm{tot}}
$$

It is necessary to emphasize that the self-energy term is not a mean-field energy of the charged chains with Gaussian statistics but the correction term caused by Gaussian correlations in the location of charges (see Appendix A for a detailed discussion). The value of the self-energy term is defined by the choice of the reference polymer system: it is the system without volume and electrostatic interactions, $C=0$ and $l_{\mathrm{b}}=0$. Therefore, the chains in the reference system possess Gaussian statistics at any length scales owing to the conformational entropy term $F_{\text {conf }}$ in the total free energy.
Thus, similar to the case of the DH plasma (eqn (23)), the correlation energy can be written as follows:

$$
\begin{aligned}
\frac{F_{\text {corr }}}{k_{\mathrm{B}} T}= & \frac{1}{2} \frac{V}{(2 \pi)^{3}} \\
& \times \int\left[\ln \left(1+\frac{1}{q^{2} \xi^{4}\left(q^{2}+r_{\mathrm{c}}^{-2}\right)}\right)-\frac{1}{q^{2} \xi^{4}\left(q^{2}+r_{\mathrm{c}}^{-2}\right)}\right] \mathrm{d}^{3} q
\end{aligned}
$$

The correlation energy (48) is always negative owing to the negative integrand, and this result fully coincides with common comprehension of correlation energy as negative energy taking into account the correlation attraction of opposite charges. In the case of an infinitely long chain, $N \rightarrow \infty$, the second term in square brackets diverges at $q \rightarrow 0$, i.e. at large distances. Indeed, the Coulomb correlation energy of an infinitely long PE chain with the Gaussian statistics at any length scales is infinite and grows with increasing chain length proportional to $\sqrt{N}$, see eqn (47). For this reason the term accounting for the chain translational entropy cannot be neglected in the total free energy.

Result similar to eqn (48) has been used earlier by Mahdi and Olvera de la Cruz who calculated the phase diagram of semidilute PE solution in the presence of salt. ${ }^{36}$ The authors justified subtraction of the second term in square brackets by mathematical convenience: this “... irrelevant subtracted term only facilitates the algebra and gives an electrostatic contribution that reduces to the simple Debye-Hückel electrostatic free energy in the correct limit." 36 In fact, subtraction of the selfenergy does not influence results for PE solution since it is a constant term and can be omitted: the self free energy density is proportional to the polymer volume fraction $\Phi$ and does not contribute to the osmotic pressure. ${ }^{70}$ However, if we would like to calculate the correlation energy of the PEC or to compare two states of the PE chains - aggregated into PEC and free - it is necessary to properly calculate and comprehend calculation of the free energy of the system. Subtraction of the self-energy of the chains is very important for distinguishing the reference state of the system and, hence, proper calculation of energy of Coulomb interactions. Application of the renormalization procedure to the case of the PEC and the proof of the negative sign of the correlation free energy are the novelties of the above part of the present work.

The self-energy is proportional to the number of PE subchains in the system $\mathscr{N}_{\text {chains }}$, like the self-energy is proportional to the number of charges in the DH plasma. The difference between these two systems is the following: point-like charges are structureless and their self-energy is unchanged under any conditions, while the PE chain can adopt different conformations resulting in different mutual spatial locations of charges and different self-energy.

The same identification of the PE chain self-energy as the energy necessary to connect charges onto a single chain has been recently used by Shen and Wang, and our choice of the reference state coincides with theirs: "the zero energy of the electrostatics is taken to be the state where charges are dispersed into infinitesimal bits at infinity." 57 The dependence 
of the chain self-energy on its conformation was discussed in ref. 57 as well. The authors introduced chain self-energy in order to find correlation free energy of PE solution, and we use very similar renormalization with the same chain self-energy definition. The only difference is that we apply it to the case of the interpolyelectrolyte complexes rather than solution of similarly charged chains.

The positive value of the PRA correction (39) means that the number of like charges surrounding any selected charge on the chain in the PEC is more than the number of opposite charges, despite repulsion of like charges and attraction of opposite charges. This fact is caused by the binding of like charges into the chain. Since these bonds exist before complex formation, their impact on the charge-charge correlations was separated from the correlations induced by purely Coulomb attraction. The Coulomb energies caused by these correlations were treated as the self-energy and the correlation energy, respectively.

\subsection{Equilibrium density of the PEC}

In order to find the equilibrium polymer volume fraction within the complex, the sum of the mean-field and the correlation free energies should be minimized. The minimization is equivalent to the equating of the osmotic pressure of the complex to zero:

$$
\Phi \frac{\partial}{\partial \Phi}\left(\frac{F_{\mathrm{MF}}+F_{\text {corr }}}{k_{\mathrm{B}} T V}\right)-\frac{F_{\mathrm{MF}}+F_{\text {corr }}}{k_{\mathrm{B}} T V}=0
$$

Here we neglect the translational entropy of polyions within the complex and the nonzero concentration of free polyions in the outer solution since their concentration is exponentially small (see Section 4 ). The RPA correction $F_{\mathrm{RPA}}$ can be used instead of $F_{\text {corr }}$ in order to find the PEC density because the self-energy does not contribute to the osmotic pressure. At that, $F_{\mathrm{RPA}}$ represents the total electrostatic energy of the system, i.e. the energy with respect to the state when all infinitesimal (not elementary) charges are moved away from each other at infinite distances. This choice of the reference state assumes that polymer chains are broken, and the energy required to gather them back into a single chain and provide the chain with the Gaussian conformation $\mathscr{U}_{\text {el-st }}^{\mathrm{G}, \mathrm{ch}}$ equals mean-field electrostatic chain energy $\mathscr{U}_{\mathrm{MF}}^{\mathrm{G} \text {,ch }}$ plus the self-energy of a chain $\mathscr{U}_{\text {corr }}^{\mathrm{G}, \mathrm{ch}}$, see Appendix A.

In the case of infinitely long chains $(N \rightarrow \infty)$, we get $F_{\mathrm{RPA}} / k_{\mathrm{B}} T V=1 /\left(6 \sqrt{2} \pi \xi^{3}\right)$, so that the polymer volume fraction within the complex reads

$$
\Phi_{\text {tot }}=\frac{\left(u f^{2}\right)^{1 / 3}}{2^{2 / 3} C^{4 / 9}(3 \pi)^{1 / 9}} \approx 0.49 u^{1 / 3} f^{2 / 3} C^{-4 / 9}
$$

and the free energy of the PEC per unit volume equals

$$
\frac{F_{\mathrm{MF}}+F_{\mathrm{RPA}}}{k_{\mathrm{B}} T V}=\frac{3^{5 / 3} u f^{2}}{4 \pi^{1 / 3} C^{1 / 3}}
$$

Thus, the free energy per chain within the macroscopic PEC phase takes the form:

$$
\mathscr{F}_{\text {coac }}=\frac{F_{\mathrm{MF}}+F_{\mathrm{RPA}}}{k_{\mathrm{B}} T \cdot \mathscr{N}_{\text {chains }}}=\frac{3^{16 / 9}}{2^{4 / 3} \pi^{2 / 9}} N C^{1 / 9} u^{2 / 3} f^{4 / 3} \approx 2.17 N C^{1 / 9} u^{2 / 3} f^{4 / 3} .
$$

Scaling analysis of the chain free energy within the PEC performed in ref. 56 predicts $\mathscr{F}_{\text {coac }} \approx-N u^{2 / 3} f^{4 / 3}$. The sign minus in the scaling result was justified by attraction of oppositely charged blobs within the complex. ${ }^{56}$ However, the negative energy of correlation attraction between oppositely charged blobs is of the order of the blob self-energy. So the scaling consideration does not allow us to define the sign (plus or minus) of the free energy within the complex, $\mathscr{F}_{\text {coac }}$. It is necessary to mention here that properly defined correlation energy of the PEC given by eqn (48) is indeed negative, but the free energy of the complex being the sum of the correlation free energy and the self-energy of the chains is positive. Moreover, scaling treats polyions as a set of disrupted blobs resulting in a linear dependence of the chain correlation energy on its length $N$, while RPA accounts for correlations within the chain on all length scales and yields asymptotically $N^{3 / 2}$.

\section{Equilibrium between non- aggregated charged chains and their complex}

In order to calculate the critical concentration of PEC formation properly, one should take into account correlation corrections to the mean-field energy of both neutral stoichiometric complex and single charged chains. Below we propose a way of calculation of the self-energy of free PE chains in the solution.

\subsection{Self-energy of a single polyelectrolyte chain}

It is well-known that the PE chain in dilute solution adopts elongated (swollen) rather than Gaussian coil conformation, Fig. 1. Owing to the small concentration of PE, all counterions are assumed to abandon the chain and distribute homogeneously throughout the solution. On the length scales $r<\xi_{\text {el-st }} \sim a u^{-1 / 3} f^{-2 / 3}$, where the electrostatic interactions do not play a role, the chain is an unperturbed Gaussian coil, while on larger scales, $r>\xi_{\text {el-st }}$, it resembles a string of the characteristic length $L \sim N a^{2} / \xi_{\text {el-st }} \sim a N u^{1 / 3} f^{2 / 3}$, Fig. 1. Thus, the intra-chain correlations are different on different length scales. Calculations of the Coulomb energy of the swollen PE chain based on the correlations predicted by scaling are performed in Appendix B.

A more rigorous method to derive the similar result for chain electrostatic energy is not to adopt a priori assumption on the blob size, $\xi_{\text {blob }}=\xi_{\text {el-st }}$. Indeed, let $\xi_{\text {blob }}$ be the size of the blob, and correlations inside it are Gaussian. The number of

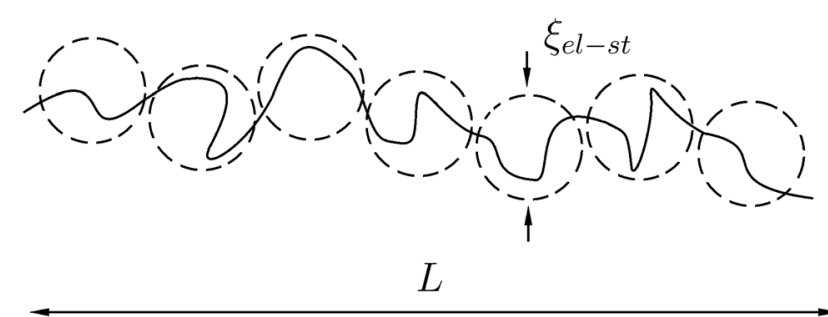

Fig. 1 Schematic representation of polyelectrolyte chain as a sequence of electrostatic blobs. 
monomer units in the blob $g \sim\left(\xi_{\text {blob }} / a\right)^{2}$ and the average distance between $i$-th and $j$-th units $\left\langle 1 / r_{i j}\right\rangle=\sqrt{6 / \pi a^{2}|i-j|}$. The electrostatic energy of a single blob is given by

$$
\frac{\mathscr{U}_{1}}{k_{\mathrm{B}} T}=\left\langle\sum \sum_{i \neq j}^{g} \frac{(e f)^{2}}{2 \varepsilon k_{\mathrm{B}} T r_{i j}}\right\rangle=u f^{2} g^{3 / 2} \sqrt{\frac{32}{3 \pi}} \simeq u f^{2} g^{3 / 2}
$$

The number of the blobs in the chain $N_{\text {blob }}=N / g=N a^{2} / \xi_{\text {blob }}{ }^{2}$. Since the PE chain is stretched, the average distance between two blobs, $k$-th and $l$-th, is given by $\left\langle r_{k l}\right\rangle=\xi_{\text {blob }}|k-l|, 1 \leq k, l \leq N_{\text {blob}}$. The charge of each blob equals $q=e f g$ and the total electrostatic energy of interactions between all blobs reads

$$
\frac{\mathscr{U}_{2}}{k_{\mathrm{B}} T}=\left\langle\sum \sum_{k \neq l}^{N_{\text {blob }}} \frac{q^{2}}{2 \varepsilon k_{\mathrm{B}} \operatorname{Tr}_{k l}}\right\rangle \simeq \frac{u f^{2} g^{2}}{\xi_{\text {blob }} / a} N_{\text {blob }} \ln \left(\frac{N_{\text {blob }}}{e}\right)
$$

Thus, the total electrostatic energy is given by

$$
\begin{aligned}
\frac{\mathscr{U}_{\mathrm{el}-\mathrm{st}}^{\mathrm{sw}, \mathrm{ch}}}{k_{\mathrm{B}} T} & =\frac{\mathscr{U}_{1}+\mathscr{U}_{2}}{k_{\mathrm{B}} T} \simeq \frac{u f^{2} g^{2}}{\xi_{\text {blob }} / a} N_{\text {blob }} \ln \left(\frac{N_{\text {blob }}}{e}\right)+u f^{2} g^{3 / 2} N_{\text {blob }} \\
& =u f^{2} g^{3 / 2} N_{\text {blob }} \ln N_{\text {blob }}
\end{aligned}
$$

while the elastic energy of deformation reads

$$
\frac{\mathscr{F}_{\mathrm{el}}}{k_{\mathrm{B}} T}=\frac{3 L^{2}}{2 N a^{2}}
$$

and $L=\xi_{\text {blob }} N_{\text {blob }}=N a^{2} / \xi_{\text {blob }}$. The total free energy of the chain can be expressed as a function of $\xi_{\text {blob }}$ which has to be minimized with respect to $\xi_{\text {blob: }}$

$$
\mathscr{F}_{\mathrm{sw}}=u f^{2} \frac{\xi_{\mathrm{blob}}}{a} N \ln \left(\frac{N a^{2}}{\xi_{\mathrm{blob}^{2}}}\right)+\frac{3 N a^{2}}{2 \xi_{\mathrm{blob}}{ }^{2}}
$$

The exact value of $\xi_{\text {blob }}$ is determined by the equation:

$$
u f^{2}\left(\ln \left(\frac{N a^{2}}{\xi_{\mathrm{blob}^{2}}}\right)-2\right)=3 \frac{a^{3}}{\xi_{\mathrm{blob}^{3}}}
$$

Taking into account $N a^{2} \gg \xi_{\text {blob }}^{2}$ and $N \gg 1$, one gets ${ }^{71}$

$$
\frac{\xi_{\text {blob }}}{a} \simeq\left(\frac{3}{u f^{2} \ln N}\right)^{1 / 3}=\left(\frac{3}{\ln N}\right)^{1 / 3}\left(u f^{2}\right)^{-1 / 3}
$$

and the total free energy can be estimated as

$$
\mathscr{F}_{\text {sw }}=\frac{3^{4 / 3}}{2}\left(u f^{2}\right)^{2 / 3} N(\ln N)^{2 / 3} \approx 2.16\left(u f^{2}\right)^{2 / 3} N(\ln N)^{2 / 3}
$$

This final result, which takes into account not only the meanfield free energy of a chain ${ }^{31,32}$ but also contribution due to the correlation of charges (see Appendix B), should be used in calculations of the critical concentration of PEC formation. The scaling estimations for the single PE chain free energy coincide with the above results up to the logarithmic and numerical factors, which are usually omitted in scaling estimations. ${ }^{56}$ However, it will be shown below that account for these corrections, 2.16 $(\ln N)^{2 / 3}$, is crucial for equilibrium between the free chains and the PEC.

A more common procedure for the self-energy calculation of a swollen PE chain in both dilute and semidilute salt solutions can be found in the recent work of Shen and Wang, where the authors took into account coupling between the chain conformation and the screening of Coulomb interactions in the solution. ${ }^{57}$ Owing to the low polyion ionization degree, $f \ll 1$, we have also neglected chain entanglements (knots), which are known to be localized, adopt a tight configuration and relax very slowly (or even remain frozen) in the case of highly charged polyelectrolytes. ${ }^{72}$

\subsection{Critical concentration of PEC formation}

In this section, we discuss the state of oppositely charged polyions in $1: 1$ solution. One can expect that in extremely dilute solutions polyions exist as single chains, while their increasing concentration should result in complexation and formation of either single globules on the basis of one polycation and one polyanion or a macroscopic condensed phase (coacervate). For the sake of simplicity, the equilibrium between the three states of the polyions, namely (i) single chains, (ii) neutral globules and (iii) macroscopic liquid coacervates will be considered, though formation of a solid precipitate is also possible in some cases, ${ }^{73}$ e.g. for oppositely charged chiral polypeptides extra capable of hydrogen bonding. ${ }^{73,74}$ Here we neglect formation of globules consisting of several couples of oppositely charged polyions assuming that their fraction will be smaller in comparison with the globules on the basis of one couple. In other words, we assume that the size distribution function of the globules has a narrow maximum for the one couple.

Let us assume that the chains are pretty long, $N\left(u f^{2} \ln N / 3\right)^{2 / 3} \gg 1$, i.e. the number of the electrostatic blobs within the chain exceeds unity by far. Free energy of the chains in each state can be divided into concentration-dependent entropic and concentration-independent energy contributions. The latter has the form:

$$
\begin{gathered}
\mathscr{F}_{\mathrm{sw}}=2.16\left(u f^{2}\right)^{2 / 3} N(\ln N)^{2 / 3} \\
\mathscr{F}_{\text {coac }}=2.17\left(u f^{2}\right)^{2 / 3} N C^{1 / 9}
\end{gathered}
$$

for the single swollen and complexed chains, respectively. Here we assume that the single PE chains do not retain counterions since energy gain provided by the trapping of one counterions is $N$ times lower than that as a consequence of PE complex formation. We also neglect the DH-type correlation attraction of these chains at an extremely small polymer concentration, which is negligible in comparison with their translational entropy, $c_{\mathrm{ch}} \ll\left(a / l_{\mathrm{b}}\right)^{3}(f N)^{-6}$. This estimation follows from the treatment of the solution as the $\mathrm{DH}$ plasma with ion charges equal to $Q=e f N$. Since the critical concentration of complex formation depends on the chain length $N$ exponentially (see below), this condition is fulfilled for long enough chains. It is also assumed that inequality $\xi \ll r_{\mathrm{c}}$ is fulfilled owing to the long enough PE chains.

The energy contribution for the case of the globule includes the energy per chain of the complex coacervate and the excess surface energy per chain, $2 \pi R_{\mathrm{gl}}{ }^{2} \gamma$. Here $\gamma a^{2} /\left(k_{\mathrm{B}} T\right)=0.11\left(u f^{2}\right)^{2 / 3} C^{-7 / 18}$ is the surface tension coefficient of the globule. ${ }^{32,75}$ This result takes into consideration connectivity of PE charges ${ }^{32}$ and for this reason differs from that found in ref. 76, where combination 
of Vroon-Overbeek and Cahn-Hilliard theories neglecting polymer specificity of the system was used. Thus, free energy per chain in globule reads

$$
\mathscr{F}_{\mathrm{gl}}=2.17\left(u f^{2}\right)^{2 / 3} N C^{1 / 9}+0.68\left(u f^{2}\right)^{4 / 9} N^{2 / 3} C^{-5 / 54} .
$$

Denoted by $M_{\text {tot }}$ the total number of polycations (polyanions) in the solution of volume $V$. Let the fraction $(1-s)$ of polyions forms coacervate, and the total number of single polycations and polycations in the globules (i.e. in supernatant) equals $M=s M_{\text {tot }}$. If all polyions in the supernatant are single chains, their free energy reads

$$
F_{0}=2 M \ln \left(\frac{M}{e V}\right)+2 M \mathscr{F}_{\mathrm{sw}}
$$

Formation of the globules can be treated as a reversible process of dimerization. Let us assume that fraction $p$ of the supernatant polyions are involved in the globules. The free energy of the supernatant can be calculated via calculation of the partition function of the system, following the approach proposed in ref. 77. The state when all chains in the supernatant are free is the reference state with the corresponding partition function $Z_{0}, F_{0}=-k_{\mathrm{B}} T \ln Z_{0}$. The partition function of the supernatant $Z$ with $p M$ globules reads

$$
Z_{\text {sn }}=Z_{0} P_{\text {comb }} W_{\text {vol }} \mathrm{e}^{2 M p\left(\mathscr{F}_{\mathrm{sw}}-\mathscr{F}_{\mathrm{gl}}\right)}
$$

$W_{\text {vol }}$ is the probability to find $M p$ polycations in the vicinity of corresponding $M p$ polyanions, namely, the center of mass of polyanions and polycations located within the volume of the order of the globule volume $v_{\mathrm{gl}}$ :

$$
W=\left(\frac{v_{\mathrm{gl}}}{V}\right)^{M p}
$$

with $v_{\text {glob }}=2 N a^{3} / \Phi_{\text {tot. }}$. The combinatorial factor $P_{\text {comb }}$ is the total number of ways to select $M p$ cations, and assemble $M p$ globules:

$$
P_{\text {comb }}=\left(\begin{array}{c}
M \\
M p
\end{array}\right)^{2}(M p) !=\frac{(M !)^{2}}{((M-M p) !)^{2}(M p) !}
$$

Thus, the free energy of the supernatant $F_{\mathrm{sn}}=-k_{\mathrm{B}} T \ln Z$ reads

$$
\begin{aligned}
F_{\text {sn }}=F_{0}+s M_{\text {tot }}[p \ln p+2(1-p) & \left.\ln (1-p)-p\left(2 \mathscr{F}_{\mathrm{sw}}-2 \mathscr{F}_{\mathrm{gl}}-1\right)\right] \\
& -s M_{\mathrm{tot}} p \ln \left(\frac{v_{\mathrm{gl}} s M_{\mathrm{tot}}}{V}\right)
\end{aligned}
$$

Finally, free energy of chains in the coacervate can be written as follows:

$$
F_{\text {coac }}=2 M_{\text {tot }}(1-s)\left[\mathscr{F}_{\text {coac }}+\ln \left(\frac{\Phi_{\text {tot }}}{2 N a^{3} e}\right)\right]
$$

The equilibrium distribution of the polyions between three states can be found via minimization of the total free energy $F_{\text {tot }}(s, p)=F_{\text {sn }}+F_{\text {coac }}$ with respect to $p$ and $s$. It has to be noted that minimization with respect to $p$ results in the law of mass action connecting concentrations of single chains and globules:

$$
\frac{p}{(1-p)^{2}}=\frac{v_{\mathrm{gl}} s M_{\mathrm{tot}}}{V} \mathrm{e}^{2\left(\mathscr{F}_{\mathrm{sw}}-\mathscr{F}_{\mathrm{gl}}\right)}
$$

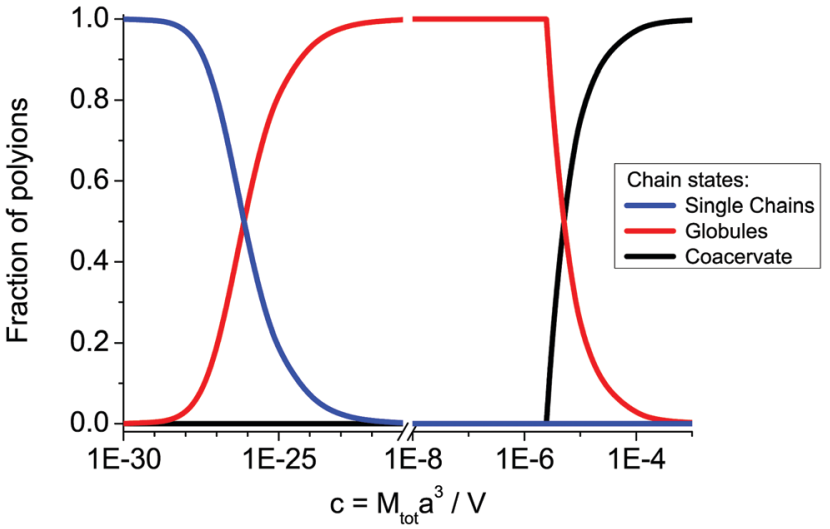

Fig. 2 Fractions of the single polyions, $s(1-p)$, complexed polyions in the globules, $s p$, and polyions in the coacervate, $(1-s)$, vs. total dimensionless concentration of polycations (polyanions) in the solution $c=M_{\text {tot }} a^{3} / V$ at $N=150, f=0.1, u=1, C=1$.

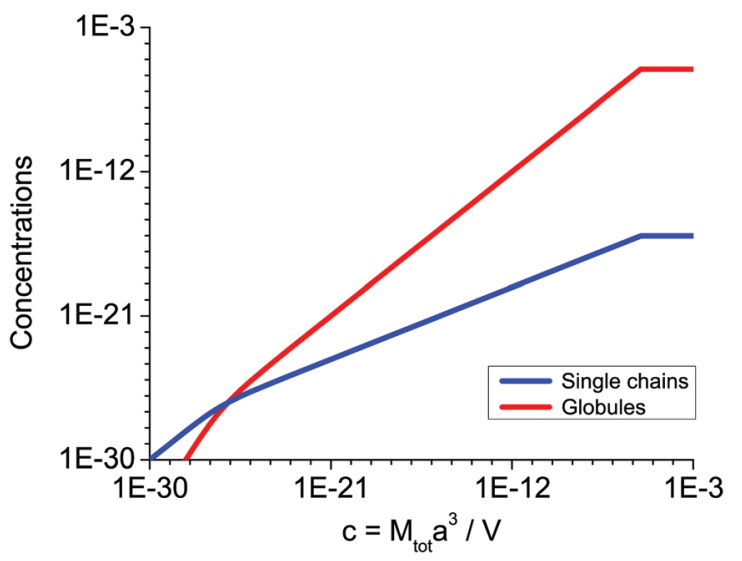

Fig. 3 Concentrations of the single polycations, $s(1-p) c$, and single globules, $s p c$, in the supernatant vs. total concentration of polycations in the solution $c=M_{\text {tot }} a^{3} / V$ at $N=150, f=0.1, u=1, C=1$.

The fractions of polyions in the coacervate, globules and non-aggregated state are shown in Fig. 2. In Fig. 3 we plot corresponding concentrations of polyions and globules in the supernatant. It is seen that increasing the polyion concentration first results in the formation of single globules. The single chains do not aggregate only at extremely small polymer concentrations. Their coexistence with the globules is observed also at extremely small polymer concentrations. The single globules dominate in the solution in a very wide concentration range, $c=10^{-20}-10^{-6}$, above which the macroscopic coacervate is formed. These numerical results coincide well with analytical estimations of the critical concentrations of the globules $c_{\mathrm{gl}}$ and coacervate $c_{\text {coac }}$ formation, which can be found from eqn (68)-(70):

$$
\begin{gathered}
c_{\mathrm{gl}} \sim \frac{1}{v_{\mathrm{gl}}} \mathrm{e}^{-2 \mathscr{\mathscr { F }}_{\mathrm{sw}}+2 \mathscr{F}_{\mathrm{gl}}} \\
c_{\text {coac }} \sim \frac{1}{v_{\mathrm{gl}}} \mathrm{e}^{-\mathscr{F}_{\mathrm{sw}}+\mathscr{F}_{\text {coac }}}
\end{gathered}
$$


Indeed, for long enough chains $c_{\mathrm{gl}} \ll c_{\text {coac }}$ as long as $2 \mathscr{F}_{\text {gl }}-\mathscr{F}_{\text {coac }}-\mathscr{F}_{\text {sw }}<0$, and the formation of the globules starts at much lower concentrations than their precipitation in the solution. At chosen values of the numerical parameters, all assumptions adopted above are valid. Namely, polyions are long enough to contain a large number of the electrostatic blobs and they exist in the non-aggregated state only at extremely small concentrations. In the concentration range shown in Fig. 2 and 3, the coacervate volume is much smaller than the total volume of the system, so that disregard of the supernatant volume diminution in the course of precipitation is justified.

Our principal result on the formation of single neutral interpolyelectrolyte globules prior to the macroscopic phase separation at increasing concentration of polyions in solution coincides with that recently reported by Delaney and Fredrickson. ${ }^{78}$ They proved this fact by means of both scaling analysis and theoretical-field methods. At that, analytical expressions for threshold concentrations, $c_{\mathrm{gl}}$ and $c_{\text {coac }}$, found in the present paper seem to be more precise as compared to scaling estimations ${ }^{78}$ where both the $\ln N$ correction as well as the surface effects were neglected, while strict theoretical-field analysis performed in their work did not allow finding analytical formulas.

\section{Conclusion}

Attraction of oppositely charged PEs within the complex is caused by fluctuation of charges and local deviation from electric neutrality. This correlation-induced attraction is opposed by repulsive volume interactions, and the equilibrium polymer volume fraction within the complex is defined from their balance. It is natural to expect that the correlation electrostatic free energy of the complex, $F_{\text {corr }}$, should be negative, similar to the case of the DH plasma. However, direct calculations of the RPA contribution, $F_{\text {RPA }}$, within the framework of a continuous model of charge distribution lead to the positive expression. This result is explained as follows. The formal calculations of the RPA Gaussian integrals take into account not only electrostatically induced correlations but also correlations caused by initial connectivity of like charges into the chain. The latter are caused by the structure of the polymer chain and should be included not into the correlation energy but into the self-energy of PE chains, $F_{\text {self }}$.

The self-energy of polyions depends on the conformation of the chain, and different chain conformations can be used as a reference state. However, in the framework of the BorueErukhimovich model ${ }^{25}$ which was widely used in the past few decades, the reference state is the Gaussian coil. This choice of the reference state is caused not only by mathematical (the structure of the free energy functional containing the conformational entropy term) but also by physical reasons. Indeed, a PEC in a $\Theta$-solvent is known to be a polymer globule, so that polyions within the globular complex/in coacervate adopt the conformation of a Gaussian coil on any length scales.

The self-energy of a PE chain within the complex equal to $\sqrt{3} u f^{2} N^{3 / 2}$ is the Coulomb energy due to the correlations of charges along the polyion in ideal coil conformation. It is important to stress that the mean-field free energy of charged polyions with Gaussian coil statistics should not be included into their self-energy because of the overall complex electric neutrality (see Appendix A). The physical meaning of the chain self-energy can be defined as follows. It is the work which is necessary to simultaneously assemble an equal number of polycations and polyanions in the same volume (volume of PEC) providing them conformations of Gaussian coil. This work is required not to get non-zero overall charge of this volume (as in the case of single polyions, eqn (60)), since the complex is electrically neutral, but to achieve connectivity of charges into Gaussian coils. In turn, energy of fluctuationinduced attraction between these already assembled polyions is the true correlation energy, $F_{\text {corr }}$.

For the first time within Borue-Erukhimovich polyelectrolyte complex model we split the RPA result for the energy of Coulomb interactions within the complex, $F_{\mathrm{RPA}}$, into two parts: self-energy of the chains, $F_{\text {self }}$, and correlation energy of the complex, $F_{\text {corr }}$. We demonstrate that the correlation free energy of the complex within the framework of this model considering continuous charge distribution, $F_{\text {corr }}=F_{\mathrm{RPA}}-F_{\text {self }}$, is negative, in accordance with general expectations. The proposed procedure for the RPA result renormalization (subtraction of the chain selfenergy) is based on physical arguments and uniquely defined. This procedure and PE chain self-energy definition are akin to those used formerly by Mahdi and Olvera de la Cruz ${ }^{36}$ and later by Shen and Wang in the case of PE solutions. ${ }^{57}$

Accounting for not only the mean-field term but also the correlation electrostatic free energy of the swollen single chain allows us to find the critical concentration $c_{\mathrm{gl}}$ of the singleglobule PEC formation and concentration of precipitation of these globules, $c_{\text {coac }}$. Analytical expressions for these concentrations are found for the case of pretty long chains providing a large number of electrostatic blobs. It is shown that the single globules of the PEC exist in a wide range of polyion concentrations, $c_{\mathrm{gl}} \ll c_{\text {coac }}$, when complexation is already favourable owing to a high energy gain in the course of polyion neutralization, while precipitation is still unfavourable because of entropic reasons.

\section{Appendix A. Mean-field and correlation energies of polyions with Gaussian statistics}

Consider a bare charged PE chain with Gaussian statistics on any length scales assuming that all counterions leave the chain. Electrostatic energy of the chain can be calculated as follows:

$$
\frac{\mathscr{U}_{\mathrm{el}-\mathrm{st}}^{\mathrm{G}, \mathrm{ch}}}{k_{\mathrm{B}} T}=\left\langle\frac{e^{2} f^{2}}{2 \varepsilon k_{\mathrm{B}} T a^{6}} \int \frac{\Phi(\mathbf{r}) \Phi\left(\mathbf{r}^{\prime}\right)}{\left|\mathbf{r}-\mathbf{r}^{\prime}\right|} \mathrm{d}^{3} r \mathrm{~d}^{3} r^{\prime}\right\rangle
$$

After substitution $\left\langle\Phi(\mathbf{r}) \Phi\left(\mathbf{r}^{\prime}\right)\right\rangle=\langle\Phi(\mathbf{r})\rangle\left\langle\Phi\left(\mathbf{r}^{\prime}\right)\right\rangle+g_{\text {chain }}\left(\left|\mathbf{r}-\mathbf{r}^{\prime}\right|\right)$, the chain electrostatic energy can be represented as a sum of two terms:

$$
\mathscr{U}_{\mathrm{el}-\mathrm{st}}^{\mathrm{G}, \mathrm{ch}}=\mathscr{U}_{\mathrm{MF}}^{\mathrm{G}, \mathrm{ch}}+\mathscr{U}_{\mathrm{corr}}^{\mathrm{G}, \mathrm{ch}}
$$


Note that the correlation function of a single Gaussian chain $g_{\mathrm{G}, \mathrm{ch}}\left(\left|\mathbf{r}-\mathbf{r}^{\prime}\right|\right)=g_{\mathrm{G}, \mathrm{ch}}(R), R=\left|\mathbf{r}-\mathbf{r}^{\prime}\right|$ coincides with $G_{\text {self }}(R)$ obtained earlier in eqn (42). This fact is a consequence of identical statistics of the single Gaussian chain and the chain in the solution of ideal (i.e. non-interacting) chains. The meanfield electrostatic energy written in the conventional form reads

$$
\frac{\mathscr{U}_{\mathrm{MF}}^{\mathrm{G}, \mathrm{ch}}}{k_{\mathrm{B}} T}=u f^{2} \int \frac{\langle\Phi(\mathbf{r})\rangle\left\langle\Phi\left(\mathbf{r}^{\prime}\right)\right\rangle}{2 a^{5}\left|\mathbf{r}-\mathbf{r}^{\prime}\right|} \mathrm{d}^{3} r \mathrm{~d}^{3} r^{\prime}
$$

and omitting numerical factor one gets $u Q^{2} / r_{\mathrm{c}}$ with $Q=N f$ being the chain charge in the elementary charge $e$ units and $r_{\mathrm{c}}=$ $a \sqrt{N} / \sqrt{12}$ being the characteristic Gaussian size of the chain. On the other hand, $\mathscr{U}_{\mathrm{el} \text {-st,MF }}^{\mathrm{G}, \mathrm{M}}=0$ within the complex since the excess charge of the $\mathrm{PE}$ molecule is compensated by the oppositely charged counterpart. The correlation term can be easily calculated:

$$
\frac{\mathscr{U}_{\text {corr }}^{\mathrm{G}, \mathrm{ch}}}{k_{\mathrm{B}} T}=\frac{2 \pi u f^{2}}{a^{6}} \int G_{\text {self }}(R) R \mathrm{~d} R \mathrm{~d}^{3} r^{\prime}=\sqrt{3} u f^{2} N^{3 / 2}
$$

This result coincides with the RPA self-energy calculations, eqn (46), and supports the proposed interpretation for the self-energy being excess Coulomb energy caused by Gaussian correlations of linked chain charges. Account for the correlations is of great importance since both the mean-field self-energy of the Gaussian chain, $\mathscr{U}_{\mathrm{MF}}^{\mathrm{G}, \mathrm{ch}}$, and the self-energy correction caused by correlations, $\mathscr{U}_{\text {corr }}^{\mathrm{G}, \mathrm{h}}$, depend on the parameters in similar ways, both $\sim u f^{2} N^{3 / 2}$. They differ only by numerical coefficients.

\section{Appendix B. Mean-field and correlation energies of the swollen polyion}

Let $\Phi_{0}$ be the average polymer volume fraction within the sphere of the diameter $\sim L$ circumscribed around the swollen PE chain (see Fig. 1), $\Phi_{0}=N a^{3} / L^{3}=\xi_{\text {el-st }}{ }^{3} /\left(N a^{3}\right)=u^{-1} f^{-2} N^{-2}$ where $\xi_{\text {el-st }} \sim a u^{-1 / 3} f^{-2 / 3}$ is the electrostatic blob. To calculate the Coulomb energy of the swollen PE chain, we use the method similar to that presented in Appendix A in the case of the Gaussian charged chain. The electrostatic energy of the chain is given by

$$
\frac{\mathscr{U}_{\mathrm{el}-\mathrm{st}}^{\mathrm{sw}, \mathrm{ch}}}{k_{\mathrm{B}} T}=\left\langle\frac{e^{2} f^{2}}{2 \varepsilon k_{\mathrm{B}} T a^{6}} \int \frac{\Phi(\mathbf{r}) \Phi\left(\mathbf{r}^{\prime}\right)}{\left|\mathbf{r}-\mathbf{r}^{\prime}\right|} \mathrm{d}^{3} r \mathrm{~d}^{3} r^{\prime}\right\rangle
$$

and $\Phi(\mathbf{r})=\Phi_{0}+\delta \Phi(\mathbf{r})$. Owing to $\left\langle\Phi(\mathbf{r}) \Phi\left(\mathbf{r}^{\prime}\right)\right\rangle=\langle\Phi(\mathbf{r})\rangle\left\langle\Phi\left(\mathbf{r}^{\prime}\right)\right\rangle+$ $\left\langle\delta \Phi(\mathbf{r}) \delta \Phi\left(\mathbf{r}^{\prime}\right)\right\rangle=\Phi_{0}{ }^{2}+G_{\text {sw }, \mathrm{ch}}\left(\mathbf{r}, \mathbf{r}^{\prime}\right)$, one can find

$$
\begin{aligned}
\frac{\mathscr{U}_{\mathrm{el}-\mathrm{st}}^{\mathrm{sw}, \mathrm{ch}}}{k_{\mathrm{B}} T} & =\frac{\mathscr{U}_{\mathrm{MF}}^{\mathrm{sw}, \mathrm{ch}}}{k_{\mathrm{B}} T}+\left\langle\frac{e^{2} f^{2}}{2 \varepsilon k_{\mathrm{B}} T a^{6}} \int \frac{G_{\mathrm{sw}, \mathrm{ch}}\left(\mathbf{r}, \mathbf{r}^{\prime}\right)}{\left|\mathbf{r}-\mathbf{r}^{\prime}\right|} \mathrm{d}^{3} r \mathrm{~d}^{3} r^{\prime}\right\rangle \\
& =\frac{\mathscr{U}_{\mathrm{MF}}^{\mathrm{sw}, \mathrm{ch}}}{k_{\mathrm{B}} T}+\frac{\mathscr{U}_{\mathrm{corr}}^{\mathrm{sw}, \mathrm{ch}}}{k_{\mathrm{B}} T}
\end{aligned}
$$

Here mean-field electrostatic energy of the chain $\mathscr{U}_{\mathrm{MF}}^{\mathrm{sw}, \mathrm{ch}}$ is defined as

$\frac{\mathscr{U}_{\mathrm{MF}}^{\mathrm{sw}, \mathrm{ch}}}{k_{\mathrm{B}} T}=\frac{e^{2} f^{2}}{2 \varepsilon k_{\mathrm{B}} T a^{6}} \int \frac{\langle\Phi(\mathbf{r})\rangle\left\langle\Phi\left(\mathbf{r}^{\prime}\right)\right\rangle}{\left|\mathbf{r}-\mathbf{r}^{\prime}\right|} \mathrm{d}^{3} r \mathrm{~d}^{3} r^{\prime} \simeq \frac{Q^{2}}{L} \simeq u^{2 / 3} f^{4 / 3} N$ since $\langle\Phi(\mathbf{r})\rangle=\Phi_{0}$ within the sphere circumscribed around the chain. The correlation electrostatic energy of the chain, $\mathscr{U}_{\text {corr }}^{\mathrm{sw}, \mathrm{ch}}$, can be found owing to its known (within the scaling consideration) correlation function, $G_{\mathrm{sw}, \mathrm{ch}}(R)$. The intra-chain correlation function is given by

$$
G_{\mathrm{sw}, \mathrm{ch}}(R)=\left\{\begin{array}{cc}
\frac{3 a \phi}{\pi R}, & R<\xi_{\mathrm{el}-\mathrm{st}} \\
\frac{3 a \phi \xi_{\mathrm{el}-\mathrm{st}}}{\pi R^{2}}, & \xi_{\mathrm{el}-\mathrm{st}}<R<L / 2
\end{array}\right.
$$

since the chain is Gaussian at $R<\xi_{\mathrm{el}-\mathrm{st}}$ and linear at $R>\xi_{\mathrm{el}-\mathrm{st}}$, and $\phi \sim u^{1 / 3} f^{2 / 3}$ is the polymer volume fraction inside the blob. Then

$$
\begin{aligned}
\frac{\mathscr{U}_{\mathrm{corr}}^{\mathrm{sw}, \mathrm{ch}}}{k_{\mathrm{B}} T} & =\frac{e^{2} f^{2}}{2 \varepsilon k_{\mathrm{B}} T a^{6}} V \int G_{\mathrm{sw}, \mathrm{ch}}(R) 4 \pi R \mathrm{~d} R \\
& =\frac{2 \pi e^{2} f^{2}}{\varepsilon k_{\mathrm{B}} T a^{6}} V\left[\frac{3 a \phi \xi_{\mathrm{el}-\mathrm{st}}}{\pi}+\frac{3 a \phi \xi_{\mathrm{el}-\mathrm{st}}}{\pi} \ln \left(\frac{L}{2 \xi_{\mathrm{el}-\mathrm{st}}}\right)\right] \\
& =6 u f^{2} \frac{\xi_{\mathrm{el}-\mathrm{st}}}{a} N \ln \left(\frac{\mathrm{e}}{2} \frac{L}{\xi_{\mathrm{el}-\mathrm{st}}}\right) \approx 6 u^{2 / 3} f^{4 / 3} N \ln \left(N u^{2 / 3} f^{4 / 3}\right)
\end{aligned}
$$

and $\mathbf{e}$ is the Euler's number. Thus, correlation electrostatic energy $\mathscr{U}_{\text {corr }}^{\mathrm{sw}, \mathrm{ch}}$ exceeds the mean-field term $\mathscr{U}_{\mathrm{MF}}^{\mathrm{sw}, \mathrm{ch}}$ by a factor of $\ln N$. For this reason account for the correlation term is requisite when charge of the chain is considered to be smeared throughout the sphere of diameter $L$.

\section{Conflicts of interest}

There are no conflicts to declare.

\section{Acknowledgements}

A. M. R. is grateful to Prof. E. Yu. Kramarenko and Prof. I. Ya. Erukhimovich for fruitful discussions. The authors acknowledge financial support from the Russian Science Foundation, project \#15-13-00124, and the Government of the Russian Federation within Act 211, contract \#02.A03.21.0011.

\section{References}

1 A. Y. Grosberg, T. T. Nguyen and I. B. I. Shklovski, Rev. Mod. Phys., 2002, 74, 329-345.

2 W. Richtering and A. Pich, Soft Matter, 2012, 8, 11423-11430.

3 R. Schroeder, A. A. Rudov, L. A. Lyon, W. Richtering, A. Pich and I. I. Potemkin, Macromolecules, 2015, 48, 5914-5927.

4 A. R. Khokhlov, J. Phys. A: Math. Gen., 1980, 13, 979-987.

5 K. N. Bakeev, V. A. Izumrudov, S. I. Kuchanov, A. B. Zezin and V. A. Kabanov, Macromolecules, 1992, 25, 4249-4254.

6 E. Spruijt, A. H. Westphal, J. W. Borst, M. A. Cohen Stuart and J. van der Gucht, Macromolecules, 2010, 43, 6476-6484.

7 Q. Wang and J. B. Schlenoff, Macromolecules, 2014, 47, 3108-3116. 
8 M. Boustta, L. Leclercq, M. Vert and V. V. Vasilevskaya, Macromolecules, 2014, 47, 3574-3581.

$9 \mathrm{~J} . \mathrm{Fu}$ and J. B. Schlenoff, J. Am. Chem. Soc., 2016, 138, 980-990.

10 A. B. Kayitmazer, D. Seeman, B. B. Minsky, P. L. Dubin and Y. Xu, Soft Matter, 2013, 9, 2552-2583.

11 F. Comert, A. J. Malanowski, F. Azarikia and P. L. Dubin, Soft Matter, 2016, 12, 4154-4161.

12 F. Comert, D. Nguyen, M. Rushanan, P. Milas, A. Y. Xu and P. L. Dubin, J. Phys. Chem. B, 2017, 121, 4466-4473.

13 E. M. Mateescu, C. Jeppesen and P. Pincus, Europhys. Lett., 1999, 46, 493-498.

14 S. Y. Park, R. F. Bruinsma and W. M. Gelbart, Europhys. Lett., 1999, 46, 454-460.

15 I. I. Potemkin, Europhys. Lett., 2004, 68, 487-493.

16 O. Lenz and C. Holm, Eur. Phys. J. E: Soft Matter Biol. Phys., 2008, 26, 191-195.

17 N. P. Shusharina, P. Linse and A. R. Khokhlov, Macromolecules, 2000, 33, 3892-3901.

18 O. V. Borisov and E. B. Zhulina, Macromolecules, 2003, 36, 10029-10036.

19 S. V. Venev, P. Reineker and I. I. Potemkin, Macromolecules, 2010, 43, 10735-10742.

20 E. A. Lysenko, A. I. Kulebyakina, P. S. Chelushkin, A. M. Rumyantsev, E. Y. Kramarenko and A. B. Zezin, Langmuir, 2012, 28, 17108-17117.

21 A. M. Rumyantsev, A. A. Rudov and I. I. Potemkin, J. Chem. Phys., 2015, 142, 171105.

22 P. Debye and E. Hückel, Phys. Z., 1923, 24, 185-206.

23 J. T. G. Overbeek and M. J. Voorn, J. Cell. Comp. Physiol., 1957, 49, 7-26.

24 V. Y. Borue and I. Y. Erukhimovich, Macromolecules, 1988, 21, 3240-3249.

25 V. Y. Borue and I. Y. Erukhimovich, Macromolecules, 1990, 23, 3625-3632.

26 J.-F. Joanny and L. Leibler, J. Phys., 1990, 51, 545-557.

27 A. R. Khokhlov and I. A. Nyrkova, Macromolecules, 1992, 25, 1493-1502.

28 A. M. Rumyantsev and E. Kramarenko, Soft Matter, 2017, DOI: $10.1039 /$ C7SM01340J.

29 M. Castelnovo and J.-F. Joanny, Langmuir, 2000, 16, 7524-7532.

30 M. Castelnovo and J.-F. Joanny, Macromolecules, 2002, 35, 4531-4538.

31 N. N. Oskolkov and I. I. Potemkin, Macromolecules, 2006, 39, 3648-3654.

32 N. N. Oskolkov and I. I. Potemkin, Macromolecules, 2007, 40, 8423-8429.

33 A. V. Ermoshkin and M. Olvera de la Cruz, Macromolecules, 2003, 36, 7824-7832.

34 A. Kudlay, A. V. Ermoshkin and M. Olvera de la Cruz, Macromolecules, 2004, 37, 9231-9241.

35 N. V. Brilliantov, Contrib. Plasma Phys., 1998, 38, 489-499.

36 K. A. Mahdi and M. Olvera de la Cruz, Macromolecules, 2000, 33, 8870-8881.

37 A. Kudlay and M. Olvera de la Cruz, J. Chem. Phys., 2004, 120, 154902.
38 I. I. Potemkin and V. V. Palyulin, Phys. Rev. E: Stat., Nonlinear, Soft Matter Phys., 2010, 81, 041802.

39 I. I. Potemkin, R. E. Limberger, A. N. Kudlay and A. R. Khokhlov, Phys. Rev. E: Stat., Nonlinear, Soft Matter Phys., 2002, 66, 011802.

40 I. I. Potemkin and A. R. Khokhlov, J. Chem. Phys., 2004, 120, 10848.

41 I. I. Potemkin, N. N. Oskolkov, A. R. Khokhlov and P. Reineker, Phys. Rev. E: Stat., Nonlinear, Soft Matter Phys., 2005, 72, 021804.

42 D. Yang, S. V. Venev, V. V. Palyulin and I. I. Potemkin, J. Chem. Phys., 2011, 34, 074901.

43 N. V. Brilliantov, D. V. Kuznetsov and R. Klein, Phys. Rev. Lett. , 1998, 81, 1433-1436.

44 N. V. Brilliantov, V. V. Malinin and R. R. Netz, Eur. Phys. J. D, 2002, 18, 339-345.

45 A. M. Tom, S. Vemparala, R. Rajesh and N. V. Brilliantov, Phys. Rev. Lett., 2016, 117, 147801.

46 Y. A. Budkov, A. L. Kolesnikov, N. Georgi, E. A. Nogovitsyn and M. G. Kiselev, J. Chem. Phys., 2015, 142, 174901.

47 M. Castelnovo and J.-F. Joanny, Eur. Phys. J. E: Soft Matter Biol. Phys., 2001, 6, 377-386.

48 J. Lee, Y. O. Popov and G. H. Fredrickson, J. Chem. Phys., 2008, 128, 224908.

49 C. E. Sing, J. W. Zwanikken and M. Olvera de la Cruz, Macromolecules, 2013, 46, 5053-5056.

50 S. L. Perry and C. Sing, Macromolecules, 2015, 48, 5040-5053.

51 S. Srivastava and M. V. Tirrel, Adv. Chem. Phys., 2016, 161, 499-544.

52 C. E. Sing, Adv. Colloid Interface Sci., 2017, 239, 2-16.

53 T. Lytle, M. Radhakrishna and C. Sing, Macromolecules, 2016, 49, 9693-9705.

54 M. Radhakrishna, K. Basu, Y. Liu, R. Shamsi, S. L. Perry and C. E. Sing, Macromolecules, 2017, 50, 3030-3037.

55 I. Borukhov, D. Andelman and H. Orland, Eur. Phys. J. B, 1998, 5, 869-880.

56 N. P. Shusharina, E. B. Zhulina, A. V. Dobrynin and M. Rubinstein, Macromolecules, 2005, 38, 8870-8881.

57 K. Shen and Z.-G. Wang, J. Chem. Phys., 2017, 146, 084901.

58 L. D. Landau and E. M. Lifshitz, Statistical Physics, Part 1, Pergamon Press, New York, 1970.

59 Z.-G. Wang, Phys. Rev. E: Stat., Nonlinear, Soft Matter Phys., 2010, 81, 021501.

60 R. Zhang and B. I. Shklovskii, Phys. A, 2005, 352, 216-238. 61 S. F. Edwards, Proc. Phys. Soc., 1966, 88, 265-280.

62 I. M. Lifshitz, Sov. Phys. - JETP, 1969, 28, 1280-1286.

63 S. B. Moldakarimov, E. Y. Kramarenko, A. R. Khokhlov and S. E. Kudaibergenov, Macromol. Theory Simul., 2001, 10, 780-788.

64 Z. Ou and M. Muthukumar, J. Chem. Phys., 2006, 124, 154902. 65 A. Salehi and R. G. Larson, Macromolecules, 2016, 49, 9706-9719. 66 A. R. Khokhlov and E. Y. Kramarenko, Macromol. Theory Simul., 1994, 3, 45-59.

67 A. G. Cherstvy, J. Phys. Chem. B, 2010, 114, 5241-5249.

68 O. E. Philippova, A. M. Rumyantsev, E. Y. Kramarenko and A. R. Khokhlov, Macromolecules, 2013, 46, 9359-9367. 
69 A. Y. Grosberg and A. R. Khokhlov, Statistical Physics of Macromolecules, AIP Press, New York, 1994.

70 J. Qin and J. J. de Pablo, Macromolecules, 2016, 49, 8789-8800.

71 P. G. de Gennes, P. Pincus, R. M. Velasco and E. J. Brochard, J. Phys., 1976, 37, 1461-1473.

72 P. G. Dommersnes, Y. Kantor and M. Kardar, Phys. Rev. E: Stat., Nonlinear, Soft Matter Phys., 2002, 66, 031802.

73 J. van der Gucht, E. Spruijt, M. Lemmers and M. A. Cohen Stuart, J. Colloid Interface Sci., 2011, 361, 407-422.

74 S. L. Perry, L. Leon, K. Q. Hoffmann, M. J. Kade, D. Priftis, K. A. Black, D. Wong, R. A. Klein, C. F. I. Piercell,
K. O. Margossian, J. K. Whitmer, J. Qin, J. J. de Pablo and M. Tirrel, Nat. Commun., 2015, 14, 6052.

75 A. S. Bodrova, E. Y. Kramarenko and I. I. Potemkin, Macromolecules, 2010, 43, 2622-2629.

76 J. Qin, D. Priftis, R. Farina, S. L. Perry, L. Leon, J. Whitmer, K. Hoffman, M. Tirrel and J. J. de Pablo, ACS Macro Lett., 2014, 3, 565-568.

77 A. N. Semenov and M. Rubinstein, Macromolecules, 1998, 31, 1373-1385.

78 K. T. Delaney and G. H. Fredrickson, J. Chem. Phys., 2017, 146, 224902. 